\title{
Real Time Embedded System for Accident Prevention
}

\author{
S. Gowtham \\ Information Technology \\ PSG Polytechnic College, Tamil Nadu, India \\ M.Sutharsan \\ Information Technology \\ PSG Polytechnic College, Tamil Nadu, India
}

\author{
S. Sethumathavan \\ Information Technology \\ PSG Polytechnic College, Tamil Nadu, India \\ Mrs. R. Uma \\ Lecturer, Information Technology \\ PSG Polytechnic College, Tamil Nadu, India
}

\begin{abstract}
This paper presents autonomous accident prevention with security enabling techniques, speed control and accident detection system. The main objective is to design an Atmega328P controller to monitor the zones, which can run on an embedded system and to automatically locate the site of accident and alert concerned people. It should be done automatically as the person involved in the accident may not be in a circumstance to send the information.The proposed system is composed of two separate design units: transmitter unit and receiver unit .Just before the vehicle is in the transmitter zone, the vehicle speed is controlled by receiving the signal from the RF transmitter. For this, RF transmitter can be kept at a few meters before the zone. Security system includes alcohol sensor.
\end{abstract}

Keywords: GSM (Global System for Mobile Communication), Alcoholsensor, vibrationsensor, LDR sensor, ultrasonic sensor.

\section{INTRODUCTION}

According to a news report, eighty percentages of road accidents are caused by human error. Based on the report of 2006 and 2012 statistics, collected from 178 participating countries, globally over 1.2 million people die in road accidents every year and 20-25 million people suffer non-fatal injuries. Pedestrians age above 65 are more than 5 times more likely to die in accidents than pedestrians age 14 or less, and the likelihood of death increases steadily for ages in between vehicle which travels at speeds above $45 \mathrm{mph}$, pedestrians above age 65 die in about 5 of 8 accidents. The main motive behind this paper is to reduce these reckless accidents for which we propose a system that controls the speed of the vehicle without any inconvenience to the driver. There are circumstances where the speed of the automobile is beyond the expected speed limit or the driver does not obey traffic signals. Therefore,we are using RFtechnology. Technology" describes about Cruise control system (CC) and Adaptive Cruise Control (ACC) which have already been developed to avoid accidents. Cruise control system is capable of maintaining predefined speed. Adaptive Cruise Control (ACC) which keeps the vehicle at pre-defined distance from the preceding vehicle systems are not able to reduce the vehicle speed automatically if driver doesn't respond. Alcohol detection and accident prevention of vehicle describes about an alcohol detecting sensor in vehicle which senses and detects alcohol gases and sends messages continuously to the relatives within every five minutes.

The position of the accident is located using Bluetooth module and android mobile phones can be detected using sensors that measure these changing parameters.There are certain parameters that change during accidents which information for help. Whenever a person sits in driver seat of a vehicle, various parameters of the system are checked. The alcohol sensor - checks if the person has consumed alcohol or not and it is suitable for detecting alcohol concentration from driver's breath. It has high sensitivity and fast response time. It provides an analog output based on alcohol concentration. If a drunk driver tries to sit on a driver seat, then the alcohol sensor MQ3 blow the buzzer and unless the alcoholic person is replaced by a normal person, the vehicle wouldn't ignite. The eye sensor ensures that the person in driver seat does not fall asleep. When the vehicle reaches the zone, it will reduce the speed. If an accident has occurred, the piezoelectric sensor provides a high value and it indicates the occurrence of an accident. Location of motor cycle is acquired using the Bluetooth module. It connects wirelessly to the mobile phone. When an accident occurs, a message will be displayed on the mobile phones having GSM.

Using state of the art LED technology makes it possible to retrain characteristics headlight icons also in low beam. The glare fare connected drive high beam assistant provides significantly improved visibility at night. The camera automatically detects the traffic situation at night and passes the information on to the headlights. Headlights adapt the light distribution according to the traffic situation.

\section{BLOCK DIAGRAM}

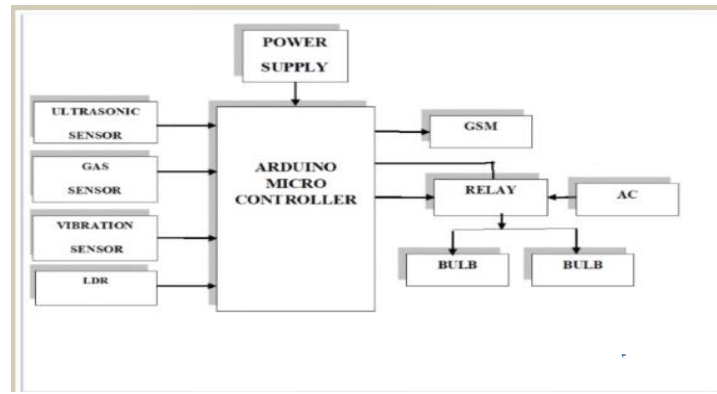




\subsection{SOFTWARE USED}

This project is implemented using Arduino IDE.

\subsection{Arduino IDE}

The software consists of a standard programming language compiler and a boot loader that executes on the microcontroller, ATmega328P. The Arduino software includes a serial monitor which allows simple textual data to be sent to and from the Arduino Uno board. The Arduino software (Arduino IDE) includes a Wire library to simplify use of the I2C bus. For Serial Peripheral Interface (SPI) communication, use the SPIbus.

\section{DESIGN PROCESS}

The RF transmitter is used for the transmission of data at the rate of 1 to $10 \mathrm{kbps}$. It will operate at a frequency range of $430 \mathrm{MHZ}$. RF transmitter is placed in specific zone and $\mathrm{RF}$ receiver is placed in the vehicle. The RF receiver also works with same frequency. The transmitted data which is sent by RF transmitter is received by the receiver and is validated. Piezoelectric sensor (vibration sensor) is connected to the port $\mathrm{A}$, which will provide analog value to the ADC port of controller.a normal person, the vehicle wouldn't ignite. The eye sensor ensures that the person in driver seat does not fall asleep. When the vehicle reaches the zone, it will reduce the speed. If an accident has occurred, the piezoelectric sensor provides a high value and it indicates the occurrence of an accidentnot.MQ6 sensor is suitable for detecting alcohol concentration from driver's breath. It has high sensitivity and fast response system checks for following parameters with the driver. The alcohol sensor checks if the person has consumed alcohol.

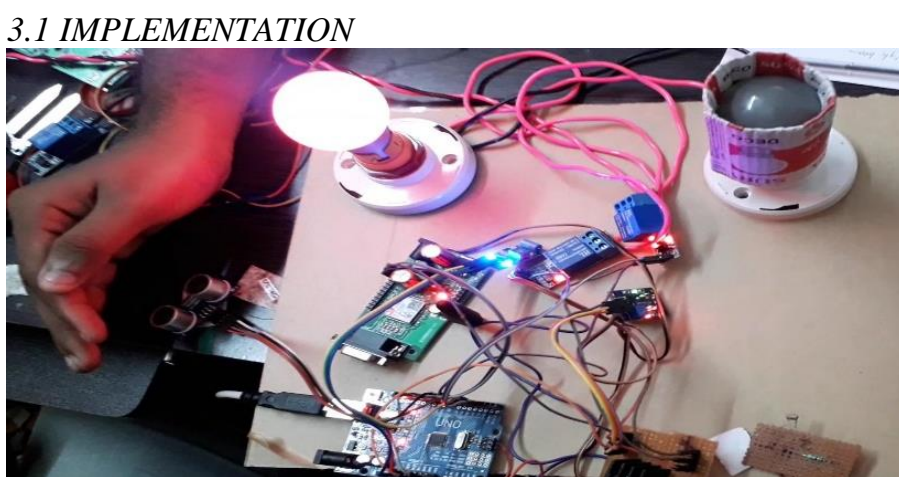

Fig 3.1LDR- Low Beam

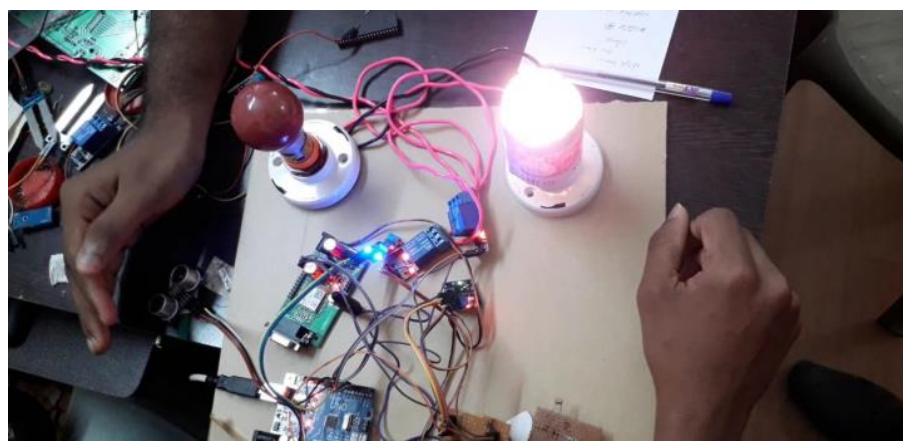

Fig 3.2 LDR- High Beam

\subsection{EXPERIMENTAL EVALUATION}

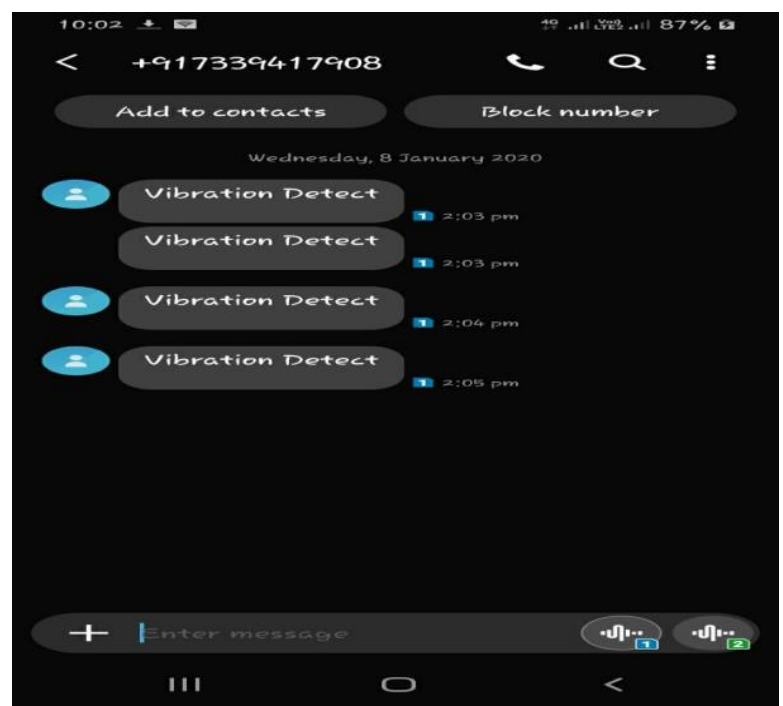

Fig 3.4 Vibration Detected

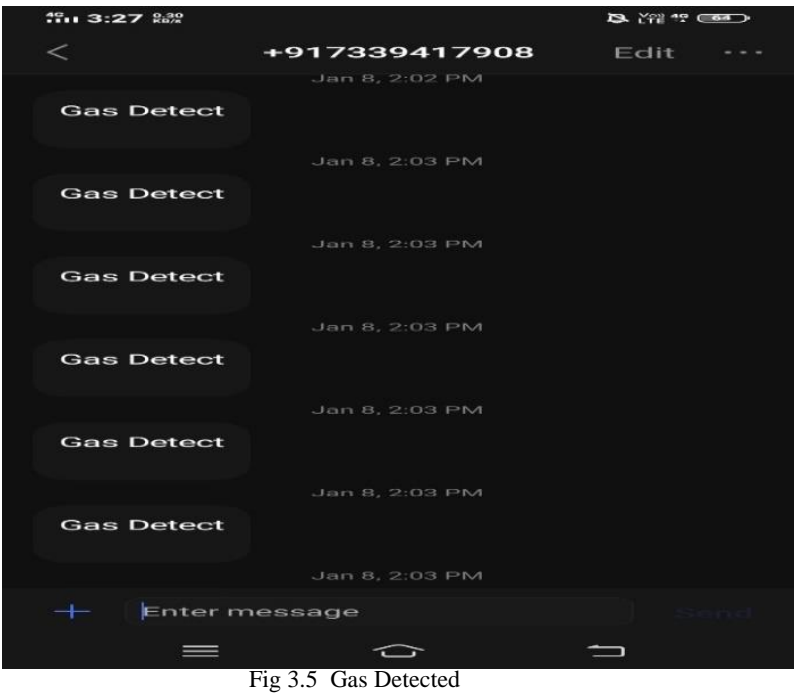

\section{CONCLUSION}

This project has been mainly designed in order to avoid accidents. It can be utilized in special areas with sudden sharp \& high curved roads. Accidents are prevented in bridges and Ghats sections. Thus, speed of the vehicle in school, work zones is reduced. Accidents due to over speed, drowsy and drunken conditions of the driver are prevented. In heavy traffic zone, speed is controlled automatically without the interference of the driver. Thus, we can reduce alcohol and drowsy related road accidents. Thus, we can bring down the alarming rate of road accidents. In night times the accident occurs because of the over intensity of the headlights and we have adjusted the headlights for the human comfort. Because of the flexibility of the embedded system, this system is compatible to any type of vehicle and is affordable to common man. 


\section{ACKNOWLEDGEMENT}

We thank Mrs.R.Uma, Faculty guide, Department of Information Technology, PSG Polytechnic College for her constant support and encouragement throughout the project period. We are grateful to have inspired guidance and valuable suggestions which motivated us right from the beginning of the project work.

\section{REFERENCES}

[1] R. Rathinakumar and D. Manivannan," Wireless accident information system using GSM and GPS," Research Journal of Applied Sciences, Engineering and Technology 4(18): 3323-3326, 2012.

[2] Mr. Sarvesh Thaware, Mr. Nilesh Pathare, Mr. Prasad Mane, Mrs. Saniya Ansari," Accident prevention and automatic speed control using eye blinking, head movement and alcohol detection", Journal of Research in Engineering and Technology, Volume 3, Issue 3, Pg. 994-998, 201

[3] Prof. R.M. Sahu, Vivek Patil, Gourav Homkar, Sachin Palve," Intelligent security system for smart vehicle," International Journalof Innovative Research in Electrical, Electronics,Instrumentation and Control engineering, Vol. 4, Issue 3, March 2016

[4] M Rajendra Prasad, P Aswani Kumar," An automated traffic accident detection and alarm device" in International Journal of Technological Exploration and Learning (IJTEL), Volume 1 Issue 1 (August 2012).

[5] C.Vidya Lakshmi, J.R. Balakrishnan,” Automatic accident detection via embedded GSM message interface with sensor technology " in International Journal of Scientific and Research Publications Volume 2,Issue 4, April 2012

[6] Ratnakar, TanmayVyas, Divyang Shah,'Automatic vehicle accident detection system based on ARM \&GPS", International Journal for Research in Technological Studies ISSN: - Applied (Online) Vol1,Issue - 1, Dec 2013

[7] Lyla B Das,"Embedded Systems" - an Integrated Approach, First Impression, Pearson, 2013

[8] Kajal Nandaniya, Viraj Choksi, AshishPatel, M B Potdar. Ph.D.," Automatic accident alert and safety system using embedded GSM interface ", International Journal of Computer Applications (0975 8887), Volume 85 - No 6, January 2014

[9] C.Prabha, R. Sunitha, R. Anita, "Automatic vehicle accident detection and messaging system using GSM and GPS modem, "International Journal of Advanced Research in Electrical, Electronics and Instrumentation Engineering Vol 3, Issue7, July 2014.

[10] Md. Syedul Amin, Jubayer Jalil," Accident detection and reporting system using GPS, GPRS and GSM Technology,” IEEE/OSA/IAPR International Conference on Informatics, Electronics \& Vision, May 2013. 